\title{
Étude De La Gestion Des Déchets Hospitaliers Dans Les Structures Sanitaires De Référence De La Région Du Nord-Cameroun
}

\author{
Justin Ndié, Inf. - Epidemiologist, Msc in Public Health \\ Ministry of Public Health, Cameroon \\ H. Blaise Nguendo Yongsi,PhD in Health Geography, Senior Lecturer \\ University of Yaoundé II, Cameroon
}

doi: 10.19044/esj.2016.v12n11p364 URL:http://dx.doi.org/10.19044/esj.2016.v12n11p364

\begin{abstract}
Hospital wastes are solid, liquid or gaseous substances which are produced as a result of diagnostic or non-diagnostic health care procedures in health facilities. These wastes generated in health facilities constitute a risk for environmental pollution and a vector for the propagation of numerous pathologies. This study was aimed at analysing the determinants of the quality of hospital wastes management in health facilities in the North Region of Cameroon. It was a cross-sectional study carried out over a period of two months ( $1^{\text {st }}$ of July to $31^{\text {st }}$ August 2015) in 13 referral health facilities of the North Region of Cameroon. A census of 12 heads of referral health facilities out of the 13 projected was made using a standardised questionnaire, in which the key items were: the political and institutional organisation, the functioning of the hospital wastes management system, the human resources, materials and finances involved in the management of the hospital wastes. The quality of the scores was calculated using points attributed to keys indicators enabling the appreciation of the level of the quality of hospital wastes management. The results of this study showed that on the political/institutional level, $41.70 \%$ of health facilities did not have a hospital hygiene unit, $66.67 \%$ did not have a reference document and no health facilities produced any report on activities of hospital wastes management. In material resources, $50 \%$ of health facilities had at least one incinerator which is more or less functional, $91.70 \%$ of health facilities had a trash can despite their non-conformity. Concerning finances, $91.70 \%$ of health facilities did not receive funds from government for hospital wastes management. In total, $92 \%$ of health facilities had a poor quality of hospital wastes management. In general, this situation is justified by the inexistence of a hospital wastes management policy. Despite certain efforts, the quality of hospital wastes management in health facilities in the North Region of
\end{abstract}


Cameroun remains low. The implementation of an operational plan which will take into account the national directives and the identified problems is necessary as it, will help in improving the quality of hospital wastes management in these health facilities.

Keywords: Hospital wastes, waste management, referral health facilities

\section{Résumé}

Les déchets hospitaliers sont des substances solides, liquides ou gazeuses issues de l'activité de soins, de diagnostic ou non, produites au niveau des formations sanitaires. Ils constituent un risque de pollution pour l'environnement et un vecteur de propagation de nombreuses pathologies. La présente étude visait à analyser les déterminants de la qualité de gestion des déchets hospitaliers dans les formations sanitaires de la Région du NordCameroun. Il s'agit d'une étude transversale descriptive réalisée pendant deux mois $\left(1^{\text {er }}\right.$ juillet au 31 août 2015) dans 13 structures sanitaires de référence de la Région du Nord. Un recensement de 12 responsables des formations sanitaires de référence sur 13 prévus a été réalisé au moyen d'un questionné standardisé, dont les principaux items étaient l'organisation politique, institutionnelle et le fonctionnement du système de gestion des déchets hospitaliers, les ressources humaines, matérielles et financières relatives à la gestion des déchets hospitaliers. Le calcul des scores de qualité par pondération simple des indicateurs a permis d'apprécier le niveau de qualité de la gestion des déchets hospitaliers. Les résultats ont montré que sur le plan politique/institutionnel, $41,70 \%$ des formations sanitaires ne disposaient pas d'une unité d'hygiène hospitalière, 66,67\% des formations sanitaires ne disposaient pas d'un document de référence et aucune formation sanitaire ne produisait les rapports d'activités de gestion des déchets hospitaliers. En ressources matérielles, 50\% des formations sanitaires avaient au moins un incinérateur plus ou moins fonctionnel, 91,70\% des formations sanitaires possédaient des seaux-poubelles malgré leur non-conformité. Sur le plan financier, 91,70\% des formations sanitaires ne recevaient pas de financement du gouvernement pour la gestion des déchets hospitaliers. Au total, $92 \%$ des formations sanitaires avaient une faible qualité de gestion des déchets hospitaliers. De manière générale, cette situation s'explique par une absence globale de politique de gestion des déchets hospitaliers. Malgré certains efforts, la qualité de gestion des déchets hospitaliers dans les structures sanitaires de la Région du Nord reste faible. La mise en œuvre d'un plan opérationnel prenant en compte les directives nationales et les problèmes identifiés est nécessaire, car il permettra d'améliorer la qualité de gestion des déchets hospitaliers dans ces formations sanitaires. 
Mots-clés : Déchets hospitaliers, gestion des déchets, structures sanitaires de référence

\section{Introduction}

Les déchets hospitaliers sont des substances solides, liquides ou gazeuses issues de l'activité de soins, de diagnostic ou non, produites au niveau des formations sanitaires. Ils présentent des risques d’infection ou non, de contamination ou d'intoxication et de pollution de l'environnement (Manga, 2009 ; Mouankié et al., 2015). De ce fait, leur gestion doit être pluridisciplinaire et s'inscrire au centre des préoccupations non seulement du politique, des collectivités mais également des formations sanitaires qui les produisent.

Selon l'OMS (2005), environ 10\% des déchets produits par les formations sanitaires sont potentiellement infectieux et dangereux. En effet, les injections par les seringues mal éliminées sont responsables de 21 millions d'hépatite $\mathrm{B}$ dont $32 \%$ de nouveaux cas, 2 millions d'hépatite $\mathrm{C}$ avec 40\% des nouveaux cas, et 260000 cas de VIH/SIDA dont 5\% des nouveaux cas (Ibid.). Bien plus, chaque année, 16 milliards d'injections sont effectuées dans le monde mais toutes les aiguilles usagées ne sont pas correctement éliminées et les déchets de soins de santé contenant des microorganismes dangereux sont susceptibles d'infecter les patients hospitalisés, les personnels de santé et le grand public (OMS, 2011). Partant, les déchets produits dans les établissements sanitaires doivent toujours suivre un itinéraire approprié et bien identifié, de leurs points de production à leur élimination finale (OMS, SCB \& PNUE, 2005).

Dans les pays en développement, de mauvaises pratiques en matière de gestion des déchets sont courantes [Ministère de la Santé du Benin (2006) et Simonsen (1999), cités par Saizonou et al. (2014)]. En Afrique, la situation reste préoccupante. En effet, l’étude menée entre 1997 et 1999 par l'Institut Africain de Gestion Urbaine au niveau de quatre villes en Afrique de l'Ouest (Bamako, Ouagadougou, Cotonou, Dakar), a montré une gestion défectueuse des déchets biomédicaux [Mbengue (1999), cité par Ndiaye et al. (2012)].

Au Cameroun, il n'existe pas encore un système standard et normalisé de traitement des déchets (Manga, 2009). Cependant, « le cadre règlementaire mis en place en matière de gestion des déchets est très peu suivi » (MINEP, 2007 : 27). Pourtant, l'ensemble des documents stratégiques notamment la Stratégie Sectorielle de la Santé (2001) et le Plan National de Développement Sanitaire (2011), prescrit comme intervention de prestation, la promotion de l'hygiène et de la gestion des déchets en milieu hospitalier. Dans les hôpitaux de première référence (hôpitaux de District) et de deuxième référence (Hôpital Régional de Garoua) de la Région du Nord- 
Cameroun, les politiques et systèmes adéquats de gestion des déchets hospitaliers sont globalement absents. Cette situation rend non effectives les étapes du processus de gestion des déchets hospitaliers et par conséquent, les déchets hospitaliers déversés dans la nature ou dans les décharges publiques par ces formations sanitaires, constituent de fait une source de pollution, de développement des maladies comme le choléra, la typhoïde, les hépatites, les dysenteries, etc. Pourtant, les responsables des formations sanitaires ont la charge d'assurer la gestion de leurs déchets (MINEP, Op. cit.). Dès lors, le problème de qualité de gestion des déchets hospitaliers se pose avec acuité ainsi que sa coordination et son suivi/évaluation dans les formations sanitaires. Ainsi, la présente étude tente d'analyser les déterminants de la qualité de gestion des déchets hospitaliers dans les structures sanitaires de la Région du Nord-Cameroun, à travers la politique, l'organisation et les ressources.

\section{Matériels et méthodes}

\section{Cadre de l'étude}

La Région du Nord est située entre $12^{\circ}-16^{\circ} \mathrm{N}$ et $7^{\circ}-10^{\circ} \mathrm{E}$. Sa superficie qui est d'environ $66263 \mathrm{~km}^{2}$ représente $14,2 \%$ de la superficie du territoire camerounais. Elle a une population d'environ 2152740 habitants (MINEPAT, 2013). Elle compte quinze districts de santé avec un Hôpital Régional ( $2^{\text {ème }}$ référence) et 12 Hôpitaux de District ( ${ }^{\text {ère }}$ référence).

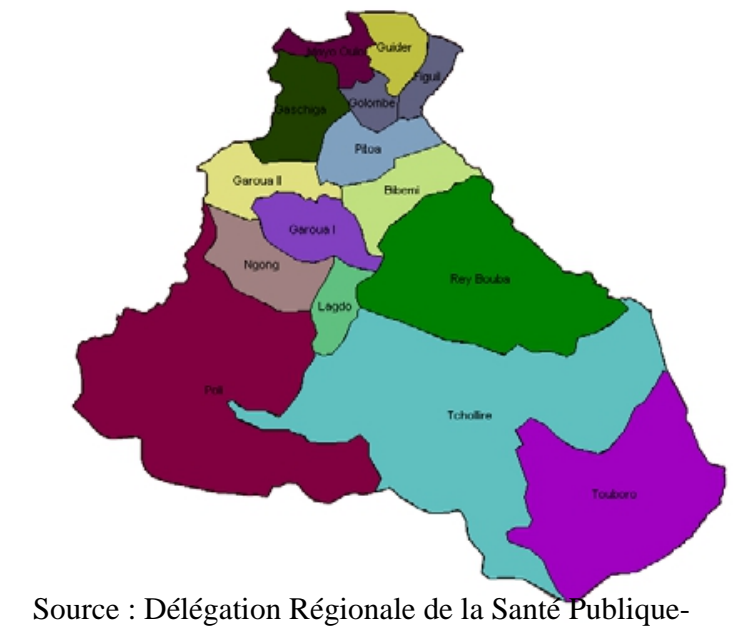

Nord, 2014

Figure 1 : Région du Nord (Découpage en District de Santé)

Nous avons mené une étude transversale descriptive du $1^{\text {er }}$ Juillet au 31 Août 2015 dans 13 structures de santé de $1^{\text {ère }}$ et de $2^{\text {ème }}$ référence dont l'Hôpital Régional de Garoua et 12 Hôpitaux de District de la Région du 
Nord-Cameroun. Étaient inclus dans notre étude les responsables des formations sanitaires ayant consenti de participer à l'étude. La technique d’échantillonnage était l'exhaustivité. Ainsi, 12 responsables ont été recensés sur 13 prévus. La collecte des données s’est faite à l'aide du questionnaire qui évaluait les déterminants institutionnels / organisationnels et politiques relatifs à la gestion des déchets hospitaliers. L’analyse des données a été effectuée à l'aide des logiciels SPSS 22, Excel 2013 et SPAD 5.

L'analyse factorielle des correspondances multiples contenant 16 variables pertinentes (indicateurs de gestion des déchets hospitaliers) a permis de dresser le profil et de regrouper les formations sanitaires de référence de la Région du Nord-Cameroun disposant ou pas de certains indicateurs de gestion des déchets hospitaliers.

\section{Variables de l'étude}

La variable dépendante de cette étude est la qualité de gestion des déchets hospitaliers. Elle est une variable polytomique ordonnée ayant pour modalité : mauvaise - acceptable - bonne. Les variables explicatives ont été regroupées en variables politiques, institutionnelles et organisationnelles. La variable dépendante est une variable score construite à partir du groupe de variables explicatives : Les variables institutionnelles/organisationnelles et politiques (Politique de gestion des déchets hospitaliers, plan de gestion de déchets hospitaliers, documents de référence, protocoles, ressources humaines, matérielles et financières, partenariat). Elles comportent 4 sousgroupes :

L’organisation/fonctionnement du système de gestion des déchets hospitaliers (organisation structurelle et organisation de l'élimination des déchets). Ces paramètres ont été déclinés en 14 indicateurs ;

Les ressources humaines (disponibilité, formation, motivation, accident). Elles comportent 5 indicateurs ;

Les ressources matérielles (disponibilité, fonctionnalité) comportant 13 indicateurs ;

comportant 3 indicateurs.

Les ressources financières (disponibilité, partenariat)

Pour l'appréciation de la qualité de la gestion des déchets hospitaliers, le calcul des scores a été effectué par pondération simple de chaque indicateur (Saizonou et al., 2014). Sur le plan descriptif, nous avons considéré que tous les indicateurs avaient le même poids, c'est-à-dire qu’ils contribuaient de manière identique à la qualité gestion des déchets hospitaliers. Ainsi, il a été attribué à chaque indicateur/critère de chaque variable explicative le score 0 pour la modalité « non » ou le score 1 pour la modalité « oui ». Ce qui définit le niveau de conformité ou non. Sur le plan analytique, le total des scores a servi de base pour apprécier chaque variable 
explicative. D’abord, le rapport entre le nombre de « oui » des critères observés sur le nombre total de « oui » des critères attendus pour apprécier le score de chaque variable ; ensuite, le total des scores a été calculé pour toutes les variables de la composante et enfin le total pour toutes les composantes pour définir le niveau de qualité de la gestion des déchets hospitaliers. Ainsi, la qualité de la gestion des déchets hospitaliers était résolue mauvaise ou faible si le score était compris 0 à $60 \%$, acceptable si le score était entre $60 \%$ et $85 \%$ et bonne si le score était compris entre $85 \%$ et $100 \%$ (Saizonou et al. Op. cit.).

\section{Résultats}

La zone d'implantation, $75 \%$ des formations sanitaires étaient implantées en zones semi-urbaine et rurale.

\section{Politique et organisation institutionnelle de la gestion des déchets}

Concernant la politique et l'organisation institutionnelle relative à la gestion des déchets hospitaliers, 58,30\% de formations sanitaires disposaient d'une unité d'hygiène hospitalière plus ou moins fonctionnelle ; 33,30\% de formations sanitaires possédaient un document de référence sur la gestion des déchets hospitaliers; 16,70\% des formations sanitaires seulement disposaient d'un plan de gestion des déchets hospitaliers; 25\% des formations sanitaires possédaient des protocoles de gestion des déchets hospitaliers parmi lesquelles 8,30\% avaient affiché les protocoles dans les services ; 66,60\% de responsables ont affirmé qu’ils ne connaissaient pas la quantité de déchets hospitaliers produite par la formation sanitaire par jour et 8,30\% formations sanitaires seulement disposaient d'un plan de suivi des déchets hospitaliers. Seulement 25\% des formations sanitaires organisaient le renforcement des capacités de leur personnel sur la gestion des déchets hospitaliers parmi lesquelles 2(16,70\%) le faisaient par occasion. En matière de supervision du personnel sur la gestion des déchets hospitaliers, 41,70\% de formations sanitaires en pratiquaient régulièrement. En revanche, seulement 16,70\% des formations sanitaires avaient été supervisés par le niveau supérieur. Par ailleurs, aucune formation sanitaire ne produisait des rapports d'activités sur la gestion des déchets hospitaliers et 91,70\% des formations sanitaires n'avaient pas de plan de suivi des activités de gestion des déchets hospitaliers.

\section{Ressources de la gestion des déchets hospitaliers Ressources humaines}

En ressources humaines, seulement 16,70\% de formations sanitaires possédaient du personnel en nombre suffisant ; 91,70\% de responsables de formations sanitaires ont affirmé que le personnel ne maîtrisait pas le 
système ou la convention des codes-couleurs des seaux/sacs poubelles; $41,70 \%$ des responsables ont déclaré que le personnel triait les déchets à la source et $83,30 \%$ ont affirmé que le personnel utilisait systématiquement les boîtes de sécurité.

\section{Ressources matérielles}

En ressources matérielles, 91,70\% disposaient de boîtes de sécurité et des seaux-poubelles malgré leur non-conformité ; 58,30\% des formations sanitaires possédaient un circuit d'acheminement des déchets hospitaliers ; 66,60\% possédaient des fosses d'enfouissement ; $91,70 \%$ de formations sanitaires disposaient d'un site de stockage des déchets hospitaliers, $50 \%$ des formations sanitaires possédaient au moins un incinérateur fonctionnel parmi lesquels $3(25 \%)$ sont de type artisanal ${ }^{12}$ et $4(33,30 \%)$ de type moderne ${ }^{13}$ et $83,30 \%$ de responsables ont déclaré que la formation sanitaire dotait le personnel des équipements de protection individuelle (EPI). En revanche, aucune formation sanitaire ne traitait les déchets hospitaliers avant leur élimination/destruction. De même, aucune formation sanitaire ne disposait d'une station d'épuration des eaux usées, ni de crématorium pour incinération les déchets anatomiques.

\section{Ressources financières}

Concernant les ressources financières, 91,70\% de responsables ont déclaré que leur formation sanitaire ne recevait pas de financement du gouvernement pour la gestion des déchets hospitaliers, 25\% des formations sanitaires avaient un partenaire financier en matière de la gestion des déchets hospitaliers parmi lesquels 2(66,67\%) avaient la Mairie comme partenaire financier et $1(8,30 \%)$ avait pour partenaire financier une ONG.

\section{Appréciation globale de la qualité de gestion des déchets hospitaliers}

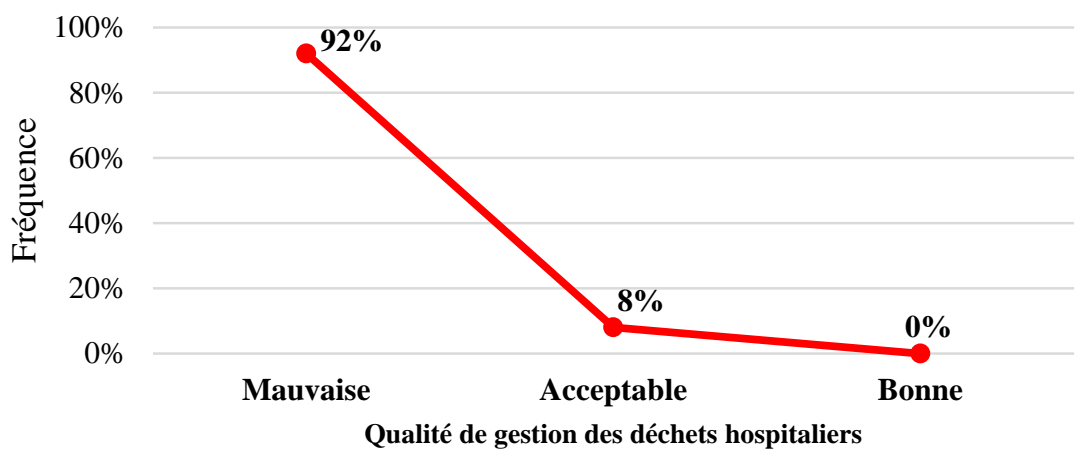

Source : données de base (pondération des indicateurs)

Figure 2: synthèse de la qualité de gestion des déchets hospitaliers

\footnotetext{
${ }^{12}$ Incinérateur fait à base de matériel local

${ }^{13}$ Incinérateur répondant à une technologie plus ou moins récente
} 
Au regard de l'appréciation des différents scores de qualité de gestion des déchets hospitaliers des formations sanitaires, 92\% des FS avaient une faible qualité de gestion des déchets hospitaliers.

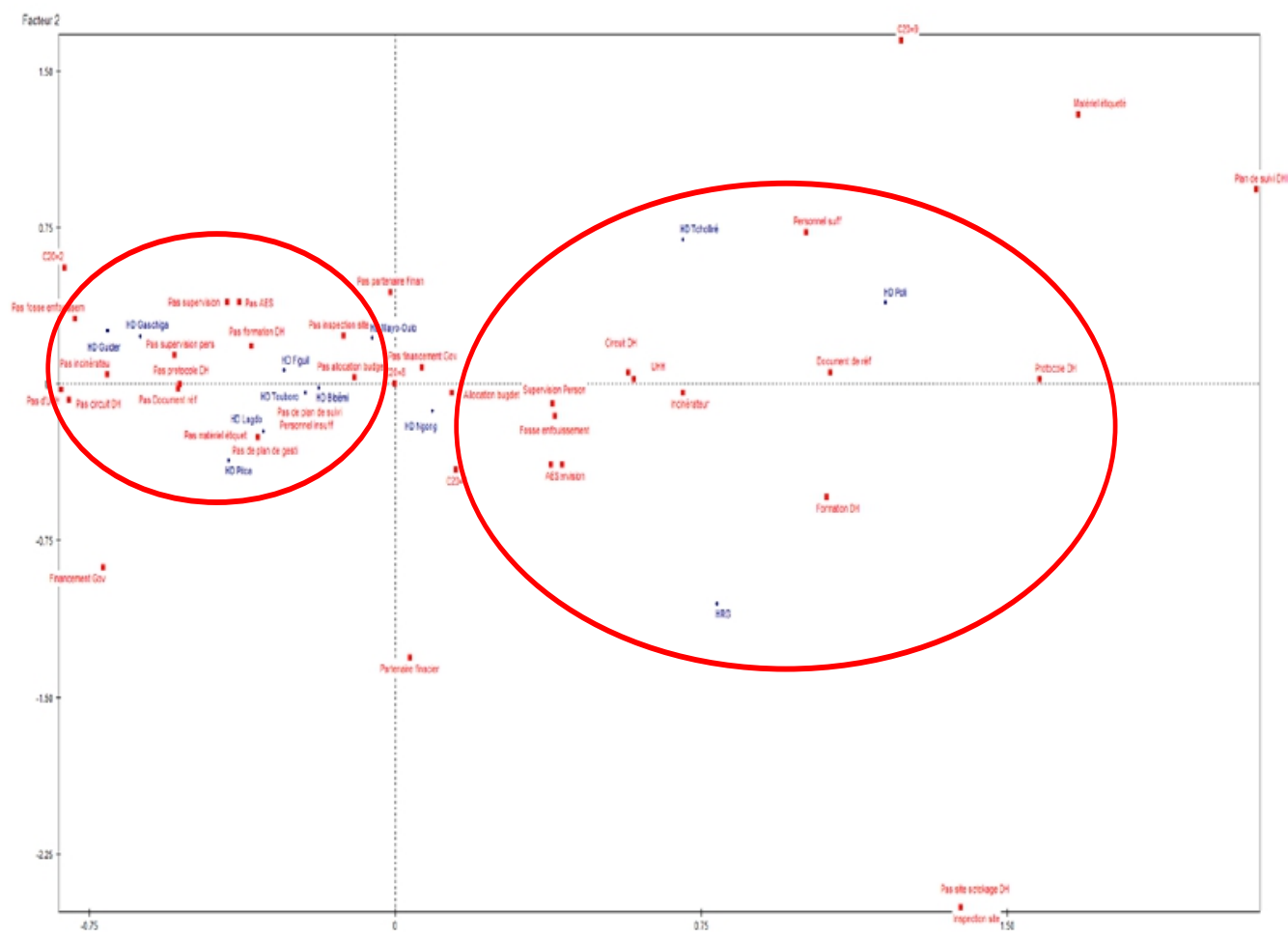

Figure 3: Carte factorielle du profil des formations sanitaires en fonction des indicateurs de gestion des déchets hospitaliers

L'Hôpital Régional de Garoua, l'Hôpital de District de Tcholliré et l'Hôpital de District de Poli sont les formations sanitaires disposant d'un minimum d'indicateurs de gestion des déchets hospitaliers notamment l'unité d'hygiène hospitalière, les protocoles, le circuit de déchets hospitaliers, la supervision et le renforcement des capacités/formation (cf. figure 3).

\section{Discussion}

\section{De la zone d'implantation des formations sanitaires}

La majorité des structures sanitaires (75\%) étaient implantées en zone rurale ou semi-urbaine. L'observation de terrain révèle que les formations sanitaires de ces zones tout comme celles des zones urbaines pour la plupart, ne disposaient pas de clôtures ou de barrières sécurisées, encore moins d'un personnel de sécurité. Cette situation laissait libre cours à la divagation des animaux domestiques et des populations dans l'enceinte de l'hôpital, mais surtout leur accès direct aux poubelles et aux sites de stockages des déchets 
hospitaliers. Notons que ces populations fouillaient régulièrement ces déchets hospitaliers et poubelles à la recherche du matériel de récupération (seringues, cartons, perfuseurs, etc.) s'exposant ainsi aux risques sanitaires (cf. photo de terrain 1).

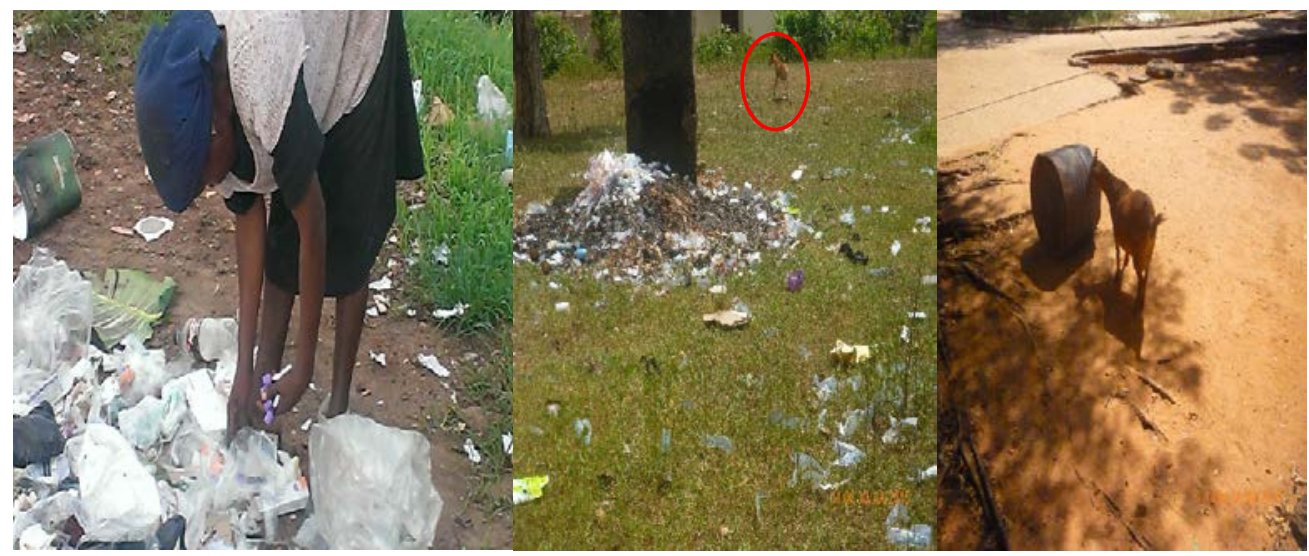

Photo de terrain 1 : Sites de décharges des déchets non sécurisés et accessibles

\section{De la politique de la gestion des déchets hospitaliers}

L'absence du document de référence sur la gestion des déchets hospitaliers a été relevée dans la majorité des structures sanitaires (66,70\%). Ces résultats sont nettement meilleurs à ceux d'Hakim (2014) au Maroc qui avait montré que la totalité d'agents d'hygiène et de gestionnaires interrogés, avaient affirmé que le document de politique de la gestion des déchets biomédicaux était inexistant dans leur structure sanitaire. Cette faible couverture des formations sanitaires en documents de référence sur la gestion des déchets hospitaliers peut être expliquée par l'immobilisme du Conseil National de l'Hygiène qui, dans un système de santé pyramidal (centralisé) a maille à élaborer un programme ou une stratégie nationale de gestion des déchets hospitaliers, de le mettre à la disposition des formations sanitaires et de le faire appliquer par l'ensemble des structures sanitaires. Pourtant, c'est un document stratégique indispensable, qui définit la politique nationale en matière de gestion des déchets hospitaliers.

\section{De l'organisation institutionnelle}

Les plans de gestion des déchets hospitaliers étaient présents dans très peu de formations sanitaires (seulement 16,70\%) ainsi que les protocoles (25\%), parmi lesquelles seule une formation sanitaire avait affiché ces protocoles dans les services. Ces résultats sont comparables à ceux de l'étude évaluative des déchets hospitaliers de l'OMS (2005) dans 22 pays dont le Cameroun, qui montraient que $18 \%$ à $64 \%$ de formations sanitaires 
n’appliquaient pas les méthodes appropriées d’élimination des déchets hospitaliers. Cet état de chose s'explique par le fait que les responsables des formations sanitaires et des unités d'hygiène hospitalière pour celles qui existent, ignoraient les standards de gestion des déchets hospitaliers d'où la rareté de ces outils essentiels dans les structures sanitaires de la Région du Nord.

À propos de l'unité d'hygiène hospitalière, une proportion non négligeable de formations sanitaires (41,70\%) n’en disposaient pas, en plus elles accusaient l'insuffisance en ressources. Ces résultats sont relativement proches de ceux de Saizonou et al. (2014) au Bénin qui montraient que l’organisation structurelle était mauvaise à 57,9\%. Cet état de chose peut être justifié par une absence de politique de gestion des déchets hospitaliers observée dans la quasi-totalité des formations sanitaires. Aussi, par la moindre importance à l'hygiène hospitalière accordée par nombre de responsables des formations sanitaires et par ricochet à la gestion des déchets hospitaliers. Davantage par l'ignorance des acteurs des risques à la fois sanitaires et environnementaux que peuvent orchestrer les déchets hospitaliers. Or, l'unité d'hygiène hospitalière est chargée de promouvoir l'hygiène hospitalière, de mettre en œuvre les politiques en la matière et de coordonner les activités y relatives. Partant, la disponibilité effective d'une l'unité d'hygiène hospitalière disposant d'un personnel qualifié dans une formation sanitaire, garantit le fonctionnement minimum du système de gestion des déchets hospitaliers. En revanche, son absence peut témoigner de la gestion anarchique des déchets hospitaliers suite à une absence de coordination voire de supervision.

La mise en œuvre effective et permanente de la supervision des activités de gestion des déchets hospitaliers permet d'identifier des manquements qui surviennent au cours de l'implémentation de cette activité et de fait, l'élaboration des stratégies correctrices et la formation illico du personnel impliqué. La supervision de la gestion des déchets hospitaliers n’était pas pratiquée dans 58,30\% des formations sanitaires et 83,30\% des formations sanitaires n’étaient pas supervisées par le niveau hiérarchique ou supérieur. La négligence de la supervision du processus de gestion des déchets hospitaliers dévolus au niveau supérieur peut être expliquée par la passivité en matière de supervision et du suivi/évaluation, mais aussi, parce que la gestion des déchets hospitaliers n'est pas inscrite régulièrement à l'agenda comme une activité prioritaire. Par conséquent, elle n’est pas prise en compte dans la planification malgré les recommandations de la Stratégie Sectorielle de la Santé (2001) et du Document de Stratégie pour la Croissance et l'Emploi (2009) du Cameroun prescrivant la promotion de l'hygiène hospitalière et de la gestion des déchets en milieu hospitalier. La rareté de supervision dans les structures sanitaires du Nord, témoigne de 
l'existence dans le processus d'élimination des déchets hospitaliers, des manquements non identifiés et non corrigés qui, forcement déteignent la qualité de gestion des déchets hospitaliers.

Dans la quasi-totalité des formations sanitaires (91,70\%) de la Région du Nord, le plan de suivi des activités de gestion des était absent. De plus, aucune formation sanitaire ne produisait les rapports d'activités relatifs à la gestion des déchets hospitaliers. Cet état de chose témoigne de la non implication des différents acteurs et de leur négligence de la gestion des déchets hospitaliers d'une part et d'autre part, la non prise en compte de cette activité par les différents plans de travail annuel. Par ailleurs, il révèle une absence notoire de traçabilité dans la gestion des déchets hospitaliers dans l'ensemble des formations sanitaires de la Région du Nord. Nonobstant, la supervision, le suivi/évaluation restent une faiblesse des formations sanitaires du Nord.

À propos du renforcement des capacités/formation, les trois-quarts des formations sanitaires (75\%) n'organisaient de renforcement des capacités / formation du personnel sur la gestion des déchets hospitaliers. Ces résultats sont similaires à ceux du PADSS-FA (2012) au Burundi, qui avaient montré que dans les différentes structures de santé, il existait très peu des capacités spécialisées dans le domaine de la gestion des déchets médicaux. Pourtant, le renforcement des capacités/formation met à jour les connaissances et les pratiques du personnel de santé. En ce sens, Nkoum (2010) observait qu'il vise à maintenir ou à améliorer les performances professionnelles.

Aucune formation sanitaire ne traitait les déchets hospitaliers avant leur élimination/destruction. De même, aucune formation sanitaire ne disposait d'une station d'épuration des eaux usées, ni de crématorium pour incinération les déchets anatomiques. Cette situation amène les acteurs à déverser les eaux usées et les déchets anatomiques potentiellement contaminées dans la nature, ce qui entraine inéluctablement la prolifération des micro-organismes et par conséquent, expose le personnel et les populations au risque d'infections nosocomiales et l'environnement à la pollution.

\section{Des ressources de gestion des déchets \\ Les ressources humaines}

Sur le plan des ressources humaines, la majorité des formations sanitaires $(83,30 \%)$ de la Région du Nord accusait une insuffisance qualitative et quantitative en personnel. Ces résultats sont similaires à ceux de l'étude d'El Maaroufi Yacine et El Ouardi El Abass (sd) au Maroc, qui montraient que la faible gestion des déchets biomédicaux était imputable à un manque de ressources humaines. Cette situation est expliquée par la pénurie en personnel de santé dans les formations sanitaires et leur mauvaise 
répartition comme l'ont relevé Mba et al. (2013). Aussi, par l'ignorance ou à la non-application par les structures sanitaires de l'arrêté $N^{\circ} 003 /$ MINEPDED du 15 Octobre 2012, fixant les conditions de gestion des déchets médicaux et pharmaceutiques qui stipule en son article 4 que tout générateur de déchets médicaux et pharmaceutiques doit mettre en place un système de gestion interne comprenant une unité chargée de gestion, un personnel qualifié et formé, un registre d'activités et un matériel adéquat. Mais davantage par le déficit d'engagement des responsables des formations sanitaires à mettre en place une unité d'hygiène hospitalière fonctionnelle et d'y nommer des responsables. Pourtant, l'OMS, SCB et PNUE (2005) recommandent qu'un responsable de la gestion des déchets hospitaliers soit nommé dans chaque établissement sanitaire et que son poste soit officiellement reconnu et tenu par des cadres supérieurs aptes à coordonner et influencer l'équipe des hôpitaux.

\section{Les ressources matérielles}

Sur le plan des ressources matérielles, la moitié des formations sanitaires (50\%) disposait au moins d'un incinérateur parmi lesquels 4(66,66\%) étaient de type moderne. Ces résultats sont le quintuple de ceux de Salifou (2007) au Bénin, qui avaient montré que 10\% des formations sanitaires de la zone sanitaire Natitingou avaient un incinérateur plus ou moins fonctionnel. Par ailleurs, ils sont nettement supérieurs à la moitié des résultats de l'étude de Saizonou et al. (2014) au Bénin qui révélait la nondisponibilité du matériel de traitement des déchets hospitaliers à 14,5\%.

Concernant le matériel de tri et conditionnement des déchets hospitaliers, une large majorité des formations sanitaires (91,70\%) disposait des boîtes de sécurité et des seaux-poubelles malgré leur non-conformité. Ces résultats sont le triple de ceux de l'enquête d'Hakim (2014) au Maroc, qui signalait la présence du matériel de conditionnement non conforme dans 33,4\% des formations sanitaires. De même, ils étaient largement supérieurs à ceux de Ngankem Ngankem II (2014) au Mali qui rapportaient que 66,7\% des services ne disposaient pas de récipients spécifiques pour la collecte des déchets hospitaliers. Dans le même sens, El Maaroufi Yacine et El Ouardi El Abass (sd) dans une étude au Maroc, montraient que le non-respect du tri des déchets était imputable à un manque de ressources matérielles.

Le circuit d'acheminement des déchets hospitaliers était absent dans $41,70 \%$ de formations sanitaires, tout comme les fosses d'enfouissement étaient absentes dans 33,40\% des formations sanitaires mais $91,70 \%$ de formations sanitaires disposaient d'un site de stockage des déchets hospitaliers. Or, d'après l'observation de terrain, ces circuits n'étaient pas connus par l'ensemble des personnes impliquées dans la gestion des déchets hospitaliers, encore moins systématiquement respectés par les 
agents d'entretien. Bien plus, ces sites de stockage des déchets hospitaliers n’étaient pas sécurisés encore moins inspectés par les experts du MINSANTE. De ce fait, les populations, les visiteurs et animaux avaient accès facile à ces sites et mêmes aux poubelles des formations sanitaires, s'exposant aux risques infectieux et traumatiques. Pourtant, l'OMS (2009) recommande la sécurisation et l'inspection des sites d'entreposage des déchets hospitaliers des formations sanitaires par le MINSANTÉ.

Par ailleurs, aucune formation sanitaire ne dispose d'un crématorium pour l'incinération des déchets anatomiques. De ce fait, les déchets anatomiques, potentiellement infectieux étaient systématiquement remis aux familles ou aux proches des patients (37,28\%). Ce qui expose davantage ceux-ci aux risques infectieux. De plus, aucune formation sanitaire ne disposait d'une station de traitement des eaux usées. Cet état de chose montre que les eaux usées sont, soit déversées dans fosses septiques, soit déversées dans la nature (33,30\%) d'où l'augmentation du risque infectieux. Aussi, aucune formation sanitaire ne traitait les déchets hospitaliers avant leur élimination/destruction. Cette situation est dû au déficit de ressources nécessaires dans les formations sanitaires.

Au regard de ce qui précède, le déficit en ressources matérielles nécessaires et adaptées à la gestion des déchets hospitaliers est prégnante dans l'ensemble des formations sanitaires de la Région du Nord. Fort de ce constat, il y a une urgente nécessité d'équiper ces structures sanitaires en ressources matérielles de pointe, essentielles à la gestion sécurisée des déchets hospitaliers. Pour ce faire, la mobilisation et la disponibilité des ressources financières s’avèrent incontournables à cet effet.

\section{Les ressources financières}

Les résultats de l'enquête de Saizonou et al. (2014) au Bénin, avaient révélé que tous les chefs de service disposaient d'un budget annuel pour la gestion des déchets biomédicaux. Ces résultats sont 58 fois supérieurs à ceux de notre enquête qui ont montré que 58,30\% de formations sanitaires n’allouaient pas de budget à la gestion des déchets hospitaliers. Par ailleurs, presque la totalité des formations sanitaires $(91,70 \%)$ ne recevait pas de financement du gouvernement pour la gestion des déchets hospitaliers. Pourtant, l'OMS, SCB et PNUE (2005) recommande le développement par chaque formation sanitaire des ressources financières spécifiques destinées à la gestion des déchets hospitaliers. Or, ces formations sanitaires n’étant pas autonomes financièrement, ce manque d'intérêt accordé à la gestion des déchets hospitaliers par les responsables restera présent. Concernant le partenariat financier ou la mobilisation des fonds, les résultats d'Hakim (2014) au Maroc, révélaient que la totalité des agents d'hygiène et gestionnaires interrogés avaient affirmé qu’il n’existait pas un mécanisme de 
mobilisation des fonds. Les résultats de notre enquête sont 25 fois supérieurs à ceux d'Hakim (2014) car 25\% des formations sanitaires avaient un partenaire financier pour la gestion des déchets hospitaliers en l'occurrence la Mairie et les ONG. L'existence d'un tel partenariat peut témoigner de la contribution des collectivités locales et des ONG à l'amélioration de la gestion des déchets. En principe, ces initiatives devraient s'étendre à toutes les formations sanitaires.

$\mathrm{Au}$ vu des résultats de l'étude, la situation qui prévaut dans les formations sanitaires de la Région du Nord se justifie par le fait de l'ignorance des outils institutionnels (lois, textes réglementaires, normes et plan national de gestion des déchets hospitaliers) et pédagogiques (formation, renforcement des capacités du personnel) relatifs à la gestion des déchets hospitaliers par les responsables des formations sanitaires et aussi par le manque de supervision du niveau supérieur.

\section{Conclusion}

Les déchets hospitaliers sont une contrepartie de l'activité de soins des établissements sanitaires. Ils restent un véritable problème de santé publique et leur gestion demeure une préoccupation du politique et des formations qui les génèrent. Malgré certains efforts, la qualité de gestion des déchets hospitaliers dans les formations sanitaires de la Région du NordCameroun reste faible (92\%). De manière générale, l'absence globale de politique et de documents de référence, le manque de ressources et la faible organisation institutionnelle du système de gestion des déchets hospitaliers sont les facteurs déterminants de la faible qualité de gestion des déchets dans les formations sanitaires de la Région du Nord-Cameroun. La mise en œuvre effective d'un système de gestion des déchets hospitaliers dans chacune des formations sanitaires de la Région du Nord-Cameroun nécessite des efforts collectifs concertés afin réduire les risques sanitaires et la pollution environnementale. Au demeurant, la gestion des déchets hospitaliers (du tri à la source jusqu'à la destruction en passant par les politiques et les ressources) doit prendre en compte les directives nationales et les problèmes identifiés et s'inscrire dans une politique globale, adaptée à la spécificité de chaque formation sanitaire. Davantage, elle doit s'inscrire dans une logique pluridisciplinaire et requiert des ressources adéquates diverses.

Au terme de cette étude, nous formulons les suggestions suivantes :

- Aux responsables des formations sanitaires, la création et la dynamisation des unités d'hygiène hospitalière chargée de mettre en œuvre la gestion des déchets hospitaliers; le renforcement des capacités du personnel et la production des rapports. 
- $\quad A u$ Délégué Régional de la Santé Publique du Nord, la mise en application effective des supervisions de la gestion des déchets hospitaliers dans les formations sanitaires ; le renforcement des capacités du personnel.

- $\quad \mathrm{Au}$ MINSANTÉ et au MINEPDED, l'élaboration de la stratégie nationale de gestion des déchets hospitaliers ; la dotation des formations sanitaires en ressources nécessaires à la gestion des déchets hospitaliers.

\section{References :}

Arrêté $\mathrm{N}^{\circ}$ 003/MINEPDED du 15 Octobre 2012, fixant les conditions de gestion des déchets médicaux et pharmaceutiques. République du Cameroun.

El Maaroufi, Y. \& El Ouardi El Abass (sd). Gestion écologique des déchets solides médicaux et pharmaceutiques : Cas de l'hôpital Avicenne de Rabat. Communication personnelle. Université Mohamed V. Rabat-Souissi / Centre Stratégique International de la Gouvernance Globale.

Faye Mbengue, M. (2008). Plan de gestion des déchets hospitaliers. Rapport. MINSANTE/DPS. République du Cameroun.

Faye Mbengue, M. (2014). Plan de gestion des déchets biomédicaux au Tchad. Rapport Final. Ministère de la Santé Publique/Ministère du Plan, de l'Économie et de la coopération Internationale. République du Tchad.

Hakim, B. (2014). Évaluation du système de gestion des déchets médicaux et pharmaceutiques " cas de l'Hôpital d'enfant de rabat ». Mémoire en Administration Sanitaire et Santé Publique. École Nationale de Santé Publique - Rabat.

Conférence Internationale sur la Prévention et le Contrôle des Infections. (2011). Session spéciale sur la sécurité des patients et la lutte contre les infections dans les services de maternité en vue de l'atteinte des OMD liés au secteur de la santé en Afrique. Genève. Suisse.

Manga, B. L. (2009). Étude sur les systèmes appropriés de traitement des déchets médicaux dans le cadre du programme national de développement participatif. Rapport du Programme National du Développement Participatif. Yaoundé - Cameroun.

Mba, R. M.; Ongolo-Zogo, P. \& Messi, F. (2013). Pour une répartition et un maintien rationnels des professionnels de santé dans les zones «difficiles d'accès »: une question d'équité dans l'offre des soins au Cameroun In Nkoum (Ed.) Tropiques Santé : Revue scientifique de l'École des Sciences de la Santé de Université Catholique d'Afrique Centrale, $n^{\circ}$ 02, PUCAC. 8199.

MINEP (2007). Stratégie Nationale de Gestion des Déchets au Cameroun (période 2007 - 2015), DRAFT V 04/09/2008. République du Cameroun. 
MINEPDED (2012). Plan national de mise en œuvre de la convention de Stockholm sur les polluants organiques persistants au Cameroun. République du Cameroun.

Ministère de l'Économie de la Planification et de l'Aménagement du Territoire. (2009). Document de Stratégie pour la Croissance et l'Emploi. République du Cameroun.

Ministère de l'Économie de la Planification et de l'Aménagement du Territoire. (2013). Rapport sur le développement économique du Cameroun. Région du Nord. République du Cameroun.

Ministère de la Santé Publique (2001). Stratégie Sectorielle de la Santé. Edition 2009. République du Cameroun.

Ministère de la Santé Publique (2011). Plan National de Développement Sanitaire. République du Cameroun.

Ministère de la Santé Publique et de la Lutte contre le SIDA/PADSS-FA (2012). Plan de gestion des déchets biomédicaux. République du Burundi.

Mouankié, J. B. et al. (2015). Gestion des déchets biomédicaux à Brazzaville capitale du Congo. European Scientific Journal. Edition vol.11, No.23 ISSN: 1857 - 7881 (Print) e - ISSN 1857- 7431. https://scholar.google.com.

Ndiaye, M. ; El Metghari, L. ; Soumah, M. M. \& Soh. M. L. (2012). Gestion des déchets biomédicaux au sein de cinq structures hospitalières de Dakar, Sénégal. Bull. Soc. Pathol. Exot. (2012) 105: 296-304 doi 10.1007/s13149012-0244-y. https://scholar.google.com.

Ngankem Ngankem II (2014). Évaluation de la gestion des déchets biomédicaux liquides dans les Centres Hospitalier Universitaires du Point G et Gabriel TOURE. Thèse de doctorat en Médecine. Université des Sciences, des Techniques et des Technologies de Bamako.

Nkoum, B. A. (2010). De l'évaluation scolaire à l'évaluation des pratiques professionnelles en santé. Paris : L’Harmattan.

OMS (2005). Gestion des déchets solides d'activités de soins dans les centres de santé primaires : Guide d'aide à la décision.

OMS. (2009). Recommandations pour améliorer la gestion des déchets médicaux.

OMS. (2011). Les déchets liés aux soins de santé. Aide-mémoire $\mathrm{N}^{\circ} 253$.

OMS-SCB \& PNUE (2005). Préparation des plans nationaux de gestion des déchets de soins médicaux en Afrique subsaharienne : manuel d'aide à la décision. Secrétariat de la Convention de Bâle et OMS.

Saizonou, J. \& al. (2014). Évaluation de la qualité de la gestion des déchets biomédicaux solides dans la zone sanitaire Klouekanme-Toviklin-Lalo au Bénin. Journal International de Santé au Travail 2014; 1 :1-11. https://scholar.google.com.

Salifou, S. (2007). Évaluation de la gestion des déchets bio médicaux solides dans les formations sanitaires de la zone sanitaire de Natitingou en 2007, au 
Bénin. Mémoire pour l’obtention du diplôme interuniversitaire. Université de Cocody d'Abidjan. 